\title{
Absorción de aceite en alimentos fritos
}

\section{Oil absorption in fried foods}

\begin{abstract}
Deep-fat frying is a cooking technique of dipping food in hot oil. Oil absorption begins with a series of structural and biochemical changes in food and oil. The final product depends on the different stages of frying. Most absorption occurs in the cooling which is the last stage of frying process. The absorption rates vary from $6 \%$, in the case of roasted nuts, until $40 \%$ with potato chips. Food composition, wetting agents, time, temperature and pre and post cooking procedures are factors that interfere with the oil absorption in frying process. Absorption rates can be diminished by pre and post frying treatments, such as the utilization of rice flour instead of wheat flour and drying by hot air, respectively. Key words: Deep-fat frying, oil absorption, fried food, frying process.
\end{abstract}

Nicolás Montes O. (1) Iván Millar M. (1)

Rosa Provoste L. (1)

Nicolás Martínez M. (1)

Débora Fernández Z. (1)

Gladys Morales I. $(1,2)$

Rodrigo Valenzuela B. (3)

(1) Carrera de Nutrición y Dietética. Facultad de Medicina. Universidad de La Frontera, Temuco, Chile. (2) Departamento de Salud Pública, Facultad de Medicina, Universidad de La Frontera, Temuco, Chile. (3) Departamento de Nutrición, Facultad de Medicina, Universidad de Chile, Santiago, Chile.

Dirigir la correspondencia a: Gladys Morales I. Departamento de Salud Pública. Facultad de Medicina. Universidad de la Frontera. Avda. Francisco Salazar 01145 Temuco, Chile.

Teléfono (56) 45-2325038; Fax (56) 45-2325000. E-mail: gladys.morales@ufrontera.cl

Este trabajo fue recibido el 16 de Marzo de 2015 y aceptado para ser publicado el 25 de Octubre de 2015.

\section{INTRODUCCIÓN}

El freír los alimentos es uno de los procesos culinarios más antiguos de los que se tiene registro, probablemente esta práctica data del siglo $\mathrm{VI}$ a. de $\mathrm{C}$, y fue probablemente uno de los primeros procesos técnico - culinarios que permitió prolongar la vida útil de los alimentos (1-2).

La fritura técnicamente corresponde a métodos de cocción de alimentos por medio de la inmersión en aceite comestible (principalmente de origen vegetal) o grasa caliente (mayoritariamente de origen vegetal o animal), por sobre el punto de ebullición del agua $\left(160-180^{\circ} \mathrm{C}\right)(3,4)$. El aceite actúa como transmisor de calor, produciendo un calentamiento rápido y uniforme del producto (2).

Las altas temperaturas durante el proceso de fritura de los alimentos causa la evaporación del agua, transfiriéndola del alimento al aceite circundante. Mientras que el aceite absorbido por el alimento reemplaza en parte el agua liberada, constituyendo hasta $40 \%$ del producto final, influenciando así todas sus propiedades organolépticas, especialmente sabor, color y aroma (4-5).

A nivel mundial, la fritura es uno de los métodos de cocción que tiene mayor aceptabilidad, lo que se ve reflejado en la amplia oferta que existe en el mercado de productos fritos y pre-fritos. Su aceptación no sólo está dada por el sabor y la textura característica de estos alimentos, sino además por la rapidez de su preparación; aspectos considerados como ventajas al utilizar esta técnica culinaria.

La Organización para la Agricultura y los Alimentos (FAO) y la Organización Mundial de la Salud (OMS) indican que el alto consumo de alimentos fritos es un factor de riesgo para la salud, principalmente por su alta densidad energética que se presenta a expensas de la grasa y por la formación de compuestos tóxicos, destacándose las acrilamidas que se han relacionado con el desarrollo de algunos tipos de cáncer (6).

Estudios epidemiológicos han informado una correlación positiva entre el incremento de la grasa de la dieta y una mayor incidencia de cáncer de mama, colon y próstata (7).

Esta revisión presenta una actualización de los diferentes factores que afectan la absorción de aceite, considerando los porcentajes de absorción en los alimentos durante el proceso de fritura. Esperamos que esta actualización sea de utilidad para profesionales del área de la alimentación, nutrición y salud, con la entrega de recomendaciones prácticas para disminuir la absorción de aceite durante el proceso de fritura.

\section{PROCESO DE FRITURA}

Gamble y cols. fueron los primeros en describir de manera razonable y científica el proceso de fritura. Durante este proceso, el vapor crea sitios de escape, mediante la búsqueda de puntos débiles en la estructura de las uniones celulares, 
acompañado de múltiples y complejas reacciones químicas que conllevan a la formación de una corteza característica del producto final (8). El proceso de fritura, actualmente se clasifica en cuatro etapas:

i) Etapa de calentamiento inicial: En esta etapa la temperatura de la superficie del alimento se eleva a temperaturas de ebullición del agua superficial. Posee una duración de 10 segundos, caracterizada por una insignificante pérdida de agua y transferencia de calor a través de convección natural.

ii) Calentamiento de la superficie: El mecanismo de transferencia de calor cambia de convección natural a convección forzada, aumentando su transferencia. Durante esta etapa del proceso, el vapor de agua liberado por el alimento, impide que el aceite ingrese. Luego, comienza la formación de la corteza de revestimiento.

iii) Etapa de velocidad decreciente: Esta etapa se caracteriza por ser la más larga de todas, donde ocurre la mayor pérdida de humedad. La temperatura del centro se acerca al punto de ebullición del agua. Posteriormente, la transferencia de vapor es constante y disminuye debido a la reducida cantidad de agua libre y el engrosamiento de la corteza, que actúa como barrera para la liberación rápida de vapor.

iv) Etapa final o "punto final de burbujeo": Esta etapa se destaca por el aparente cese de la pérdida de humedad en los alimentos, pudiendo deberse a la falta de agua líquida o una reducción en la transferencia de calor en la interfaz de la corteza/centro. La conductividad térmica de la corteza es baja debido a su sequedad y porosidad. Cabe destacar, que la absorción continúa luego de retirar el producto del aceite $(9,10)$.

En la figura 1 se presenta el proceso de fritura.

\section{FRITURA Y ABSORCIÓN DE ACEITE}

Para establecer la cantidad de aceite absorbido durante el proceso de fritura, primero se debe comprender cómo se absorbe el aceite en los alimentos fritos. Dana y cols. (11) se- ñalaron tres mecanismos: Agentes tensioactivos, reemplazo de agua y efecto en la fase de enfriamiento. No obstante, Mellema (5) concuerda con estos dos últimos, los cuales se describen a continuación:

i) Mecanismo de reemplazo de agua: Durante la fritura se produce una transferencia de calor entre el alimento y el ambiente. El agua se evapora rápidamente y la superficie exterior se seca, formando una costra por la existencia de dos regiones en constante movimiento, una deshidratada denominada corteza y un centro húmedo. La humedad en el producto frito se convierte en vapor, creando una gradiente de presión positiva, esto determina que el vapor escape por las grietas y abra los capilares (canales en la estructura y en las membranas celulares), generando que durante esta fase exista un menor ingreso de aceite, el cual corresponde aproximadamente a $20 \%$ de la absorción final. Sin embargo, la absorción del aceite no se produce si los poros de la superficie del alimento están siendo ocupados por el vapor de agua. Por lo tanto, la absorción de aceite está determinada en gran medida por el contenido de humedad de los alimentos (2,5,9,11-12).

ii) Fase de enfriamiento o condensación: Al retirar el alimento del aceite caliente, el núcleo de este comienza a enfriarse, provocando la condensación gradual del vapor de agua presente en el interior del alimento. En consecuencia, disminuye la presión interna que provoca un efecto de vacío, donde se produce la mayor absorción y el aceite adherido a la superficie del alimento es aspirado (2,5,9,11-15).

Moreira (12), en un estudio realizado en tortillas, encontró que la mayor cantidad de aceite ingresa en esta fase, determinando que $64 \%$ del aceite superficial de post fritura se absorbe, y el restante queda alojado en la superficie.

\section{FACTORES QUE AFECTAN LA ABSORCIÓN DE ACEITE}

Existen múltiples factores que afectan el proceso de absorción de aceite, los cuales se describen a continuación: i) Temperatura: No tiene ningún efecto significativo entre

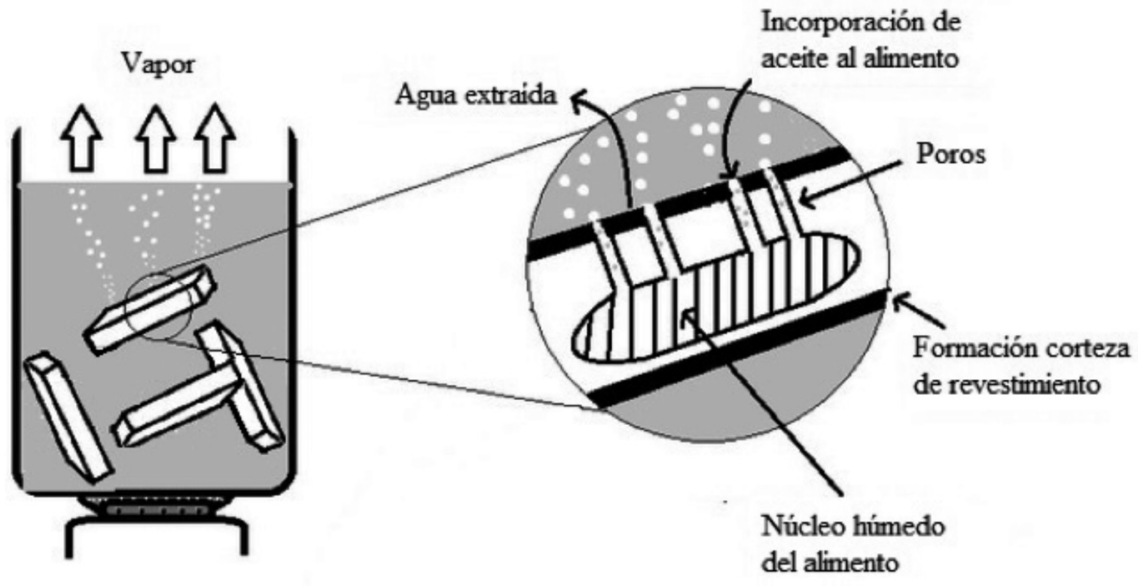


$150^{\circ} \mathrm{C}$ y $180^{\circ} \mathrm{C}$. Cuando mayor sea la temperatura, menor será la absorción de aceite en la superficie, y por el contrario, un exceso en la absorción de aceite puede ser consecuencia de bajas temperaturas (16). Esto concuerda con lo señalado por la FAO/OMS, que la fritura en aceite debe mantenerse a una temperatura máxima de $180^{\circ} \mathrm{C}$ (17). Altas temperaturas (a partir de los $200^{\circ}$ ) aceleran el proceso de fritura y también la descomposición del aceite utilizado (18).

ii) Tiempo: El tiempo de fritura es otro factor principal que aumenta la absorción de aceite. Se encontró una correlación entre tiempo y temperatura al momento de freír, por lo tanto, es común encontrar que a mayor temperatura menor es el tiempo de fritura (18-19). Un incremento en el tiempo de fritura, produce una mayor alteración del aceite y del alimento que se fríe. Además el tiempo del calentamiento del aceite disminuye su estabilidad y se producen diferentes compuestos tóxicos (20).

iii) Características del alimento: La composición de los alimentos, en particular, la estructura de la superficie, la humedad, el contenido de lípidos, la forma del producto, la relación superficie/peso y la porosidad, son características del alimento que afectan a los procesos de pérdida de agua y absorción de aceite. (19)

Sumado a lo anterior, se ha considerado la rugosidad superficial como otra característica importante que interviene en el aumento de la absorción de aceite. Un aumento del área de contacto del alimento con el aceite, tendrá una mayor incidencia en la absorción durante la fritura (2).

iv) Agentes humectantes: Durante la fritura el aceite es degradado, cambiando dinámicamente su composición a una mezcla de compuestos polares (diglicéridos, monoglicéridos, ácidos grasos libres y glicerol). Estos compuestos de degradación actúan como agentes humectantes, que sirven para reducir la tensión superficial entre aceite y agua, aumentando la absorción del aceite. A mayor cantidad de agentes humectantes liberados se provoca un aumento en la viscosidad, aumentando la cantidad de aceite que se adhiere a la superficie del alimento. Además la tensión interfacial entre el alimento y el aceite disminuye, facilitando de esta manera su absorción (9).

La calidad del aceite es un aspecto importante a considerar en la formación de compuestos de degradación. Los aceites de alto contenido de ácido oleico presentan mayor estabilidad en su estructura química al someterlos a altas temperaturas, entre estos se destacan el aceite de oliva y aceites modificados genéticamente como el de girasol y cártamo "alto oleico" $(17,20,21)$.

Es importante destacar que existen diversas ventajas al freír con aceite de oliva, en particular el extra virgen en comparación con otros aceites (22), como las que se mencionan a continuación:

- La composición del aceite presenta un alto porcentaje de ácidos grasos monoinsaturados (ácido oleico). Además presenta un alto aporte de compuestos fenólicos, que son antioxidantes.

- Menor cantidad de compuestos polares, hidroperóxidos y otros compuestos de alteración secundaria (polímeros, dímeros).

- Mejor textura y palatabilidad de los alimentos fritos. Además previene el exceso de absorción de grasa.

v) Tratamientos pre y post- fritura: Diversos estudios han señalado que existen muchos tratamientos de pre fritura que intentan reducir la absorción mediante la reducción de la permeabilidad de la superficie. Esto puede lograrse a través de una capa comestible o directamente una modificación de la mezcla, como en productos formulados, usando hidrocoloides como metilcelulosa, hidropropilmetilcelulosa, fibras largas de celulosa, zeína de maíz, almidón modificado, entre otros $(4-5,23)$. Thanatuksorn y cols. plantearon que la harina modificada por un tratamiento de molienda tiene poros más grandes y menor contenido de humedad, por lo que hay menor penetración de aceite al final del proceso (24), destacando que las masas hechas de harina de arroz de grano largo retienen menos aceite durante la fritura que las masas hechas de harina de trigo. El utilizar agentes espesantes a base de arroz como aditivos, es una buena estrategia para disminuir la absorción de aceite, mejorar la consistencia y viscosidad de las masas hechas de harina de arroz de grano largo. Particularmente la harina de arroz de grano largo gelatinizada y ésteres de almidón de arroz de grano largo fosforilados, pueden ser eficaces en la mejora de las propiedades reductoras de la absorción de aceite, debido a la viscosidad de esta masa (25). Se ha demostrado que la absorción de aceite es 50\% menor en alimentos rebozados en base a harina de arroz, en comparación con harina de maíz o trigo. Además se encontró que la formación de acrilamida era tres veces mayor en este último caso,(25-26).

Los tratamientos de post fritura como el secado por aire caliente y el secado por vapor muy caliente, son dos procesos que han mostrado reducir significativamente la absorción de aceite después de la fritura. Se reduce bajando el contenido de humedad del alimento antes de freír, usando horno microondas, horno convencional y aire caliente $(4,27-28)$. Moreno y Bouchon, señalaron que los alimentos secados por aire disminuyen la absorción de aceite por la baja permeabilidad de la capa externa,(4).

\section{PORCENTAJES DE ABSORCIÓN DE ACEITE}

La absorción de aceite puede variar desde $6 \%$, en el caso de los frutos secos tostados, hasta $40 \%$ aproximadamente en el caso de las papas fritas (19). Mientras que los alimentos rebosados en base a harina (pescado o pollo) absorben aproximadamente $15 \%$ de aceite de fritura, mientras que el pescado o pollo rebosado en base a pan lo hace en $20 \%$. La cantidad de aceite absorbido por las masas dulces varían entre 15 a $20 \%$ de su peso final, sin considerar la manteca o aceite utilizado en la preparación, quedando entonces hasta 30\% de contenido de grasa final. Las papas fritas en forma de "bastón" absorben en promedio $10 \%$ de su peso total en aceite. Las papas fritas "chips" absorben la mayor cantidad de aceite; variando entre 35 a 40\%. Se han introducido al mercado papas fritas bajas en grasa, las que contienen aproximadamente $20 \%$ de aceite. Por lo tanto, hay que recordar que el aceite utilizado para freír se convierte en parte de los alimentos que se consumen (29).

\section{FRITURA, ABSORCIÓN DE ACEITE Y SALUD}

Hoy en día los países desarrollados y también los en vías de desarrollo enfrentan una creciente pandemia de sobrepeso y obesidad, a la cual algunos investigadores le han dado el nombre de "Globesidad" (obesidad global) (30), considerando que más de mil millones de personas presentan malnutrición por exceso.

Existen diversas razones a esta problemática, una de ellas es el excesivo consumo de alimentos, puesto de manifiesto por la Encuesta Nacional de Consumo Alimentario (ENCA) 2010-2011, que destaca un consumo excesivo de energía, grasas saturadas, azúcares y sodio en todos los grupos etarios, 
especialmente en los niveles socioeconómicos más bajos.

En relación a las grasas, las más consumidas fueron las saturadas con 52,8\% (31). Por lo anteriormente señalado, se destaca que los alimentos fritos son una importante fuente de calorías y grasas, específicamente de ácidos grasos saturados (AGS) y ácidos grasos trans (AGT) $(32,33)$. Diversos estudios han mostrado la asociación entre ingesta de ácidos grasos en la dieta, niveles de colesterol sérico y las enfermedades cardiovasculares (34). En estudios metabólicos se ha evidenciado que los AGS aumentan los niveles de colesterol (35). Sin embargo, se ha demostrado que este efecto depende del tipo de ácido graso. Los efectos deletéreos de los AGS han sido descritos sólo para los ácidos laúrico (12:0), mirístico (14:0) y palmítico (16:0) (36); siendo el más potente el mirístico (37)

Se ha demostrado que el consumo de AGT eleva el riesgo de ECV, muerte súbita y el riesgo de diabetes tipo 2. Esta asociación es más fuerte que la observada con AGS (38). Los AGT modifican negativamente el perfil lipídico, aumentando los niveles de colesterol LDL y reduciendo los niveles de colesterol HDL sanguíneos. Además, se han asociado a altos niveles de marcadores inflamatorios circulantes como interleuquina - 6 y proteína $C$ reactiva (39). De acuerdo a la recomendación establecida por el comité de expertos OMS-FAO sobre la ingesta de AGS, no debe exceder $10 \%$ de la energía diaria y la ingesta de AGT debe ser menor del 1\% de la energía total, para lograr la prevención de ECV (36).

Por todo lo anteriormente señalado, en la tabla 1, se señalan algunas recomendaciones prácticas para disminuir la absorción de aceite durante el proceso de fritura y lograr un alimento frito menos nocivo para la salud.

\section{CONCLUSIONES}

La absorción de aceite ocurre durante y posterior al proceso de fritura. Durante la etapa de enfriamiento ocurre el mayor porcentaje de absorción, debido al proceso de condensación de la humedad del alimento, dejando poros por donde ingresa el aceite. La temperatura, tiempo, composición de los alimentos, agentes humectantes y tratamientos culinarios de pre y post-fritura, son los principales factores que intervienen en la absorción de aceite durante el proceso de fritura. Los tratamientos de pre-fritura como la adición de hidrocoloides, almidón modificado y utilización de harina de arroz, en reemplazo de la harina de trigo, junto a los tratamientos de postfritura como el secado por aire caliente y vapor muy caliente, han mostrado reducir significativamente la absorción de aceite. En relación a los porcentajes de absorción de aceite en los distintos tipos de alimentos, durante el proceso de fritura, existe poca información disponible. Sin embargo existe claridad que las papas fritas chips son las que absorben la mayor cantidad de aceite, en comparación con otros tipos de alimentos fritos, como alimentos apanados, masas y frutos secos.

\section{RESUMEN}

Freír es una técnica culinaria por inmersión de alimentos en aceite caliente. La absorción de aceite comienza con una serie de transformaciones estructurales y bioquímicas del alimento y del aceite. Las distintas etapas del proceso de fritura afectan el producto final. En la última etapa, de enfriamiento, se produce la mayor absorción. Los porcentajes de absorción varían de $6 \%$ en el caso de los frutos secos tostados, hasta $40 \%$ en el caso de las papas fritas. La temperatura, tiempo, composición de los alimentos, agentes humectantes y tratamientos culinarios de pre y post fritura, son los principales factores que interfieren en la absorción de aceite durante este proceso. Se ha demostrado que la utilización de harina de arroz en reemplazo de la harina de trigo y el secado por aire caliente, son tratamiento de pre y post fritura respectivamente, que han logrado reducir los porcentajes de absorción de aceite.

Palabras clave: Freír, absorción de aceite, alimento frito, proceso de fritura.

Agradecimientos: Los autores agradecen a la Srta. Anita Cotal, Srta. María José González y Srta. Lesly Zambrano, integrantes de la Sociedad Científica de Estudiantes de Nutrición de la Universidad de la Frontera (SOCENUT), por su valioso aporte en la presente revisión bibliográfica.

\section{TABLA 1}

Recomendaciones prácticas para disminuir la absorción de aceite durante el proceso de fritura y obtener un alimento frito menos nocivo para la salud.

1. Utilizar un cuchillo liso para cortar los alimentos que se van a freír (ej. Papas fritas), de tal manera que la superficie del alimento sea lo más lisa posible (5).

2. Utilizar aceites con mayor contenido de ácido oleico, como el aceite de Oliva o girasol "alto oleico" (11), porque son más estables a altas temperaturas.

3. Secar los alimentos tanto frescos como congelados y pre-fritos antes de que sean fritos. Ej. En el horno tradicional o microondas $(4,25-26,40-41)$ o bien con papel absorbente.

4. Al momento de extraer el alimento de la fritura, se debe agitar intensamente, procurando drenar la mayor cantidad de aceite posible (5)

5. Al rebozar alimentos, utilizar espesantes en base a harina de arroz $(26,41)$.

6. Al terminar el proceso de fritura, poner el alimento en papel absorbente para eliminar el exceso de aceite $(4,11)$.

7. No reutilizar el aceite para freír, evitando frituras discontinuas. Debido a que repetidos calentamientos y enfriamientos, incrementa los compuestos tóxicos, como hidroperóxidos y radicales libres $(2,42)$. 


\section{BIBLIOGRAFÍA}

1. Morton I. Geography and history of the frying process. II Symposium Internacional sobre "fritura de los alimentos", Madrid (España), Consejo Superior de Investigaciones Científicas. 1996; 49: 247-9.

2. Dana $D$, Saguy S. Integrated approach to deep fat frying: engineering nutrition, health and consumer aspects. J Food Eng. 2003; 56:143-52.

3. Farkas $B$, Singh $R$, Rumsey $T$. Modeling heat and mass transfer in immersion frying. I Model development. J Food Eng. 1996; 29(2):211-26.

4. Moreno M, Bouchon P. A different perspective to study the effect of freeze, air, and osmotic drying on oil absorption during potato frying. J Food Sci. 2008; 73:122-8.

5. Mellema M. Mechanism and reduction of fat uptake in deep-fat fried foods. Trends Food Sci Technol. 2003; 14(9):364-73.

6. FAO/OMS. Consecuencias para la salud de acrilamidas en alimentos. Ginebra. 2002.

7. BNF (British Nutrition Foundation) report. Trans fatty acids. London: BNF. 1887.

8. Gamble $M H$, Rice P, Selman JD. Relationship between oil uptake and moisture loss during frying of potato slices from C.V. Record U.K. tubers. Int J Food Sci Tech. 1987; 22:233-41.

9. Brannan R, Mah E, Schott M, Yuan S, Casher K, Myers A $y$ cols. Influence of ingredients that reduce oil absorption during inmersion frying of battered and breaded foods. Eur J Lipid Sci Technol. 2014; 116:240-54.

10. Mehta U. Swinburn B. A Review of Factors Affecting Fat Absorption in Hot Chips. Crit Rev Food Sci Nutr. 2001; 41:2, 133-54.

11. Dana D, Saguy S. Review: Mechanism of oil uptake during deep-fat frying and the surfactant effect-theory and myth. Adv Colloid Interface Sci. 2006; 128-130:267-72.

12. Moreira RG, Sun $X$, Chen $Y$. Factors affecting oil uptake in tortilla chips in deep-fat frying. J Food Eng. 1997; 31:485-98.

13. Bouchon P, Aguilera JM, Pyle DL. Structure oil-absorption relationships during deep-fat frying. J Food Sci. 2003; 68:2711-6.

14. Cortés P, Badillo G, Segura L, Bouchon P. Experimental evidence of water loss and oil uptake during simulated deep-fat frying using glass micromodels. J Food Eng. 2014; 140:19-27.

15. Ufheil G, Escher FG. Dynamics of Oil Uptake during Deepfat Frying of Potato Slices. Lebensm Wiss Technol. 1996; 29:640-4.

16. Varela G. Les graisses chauffées: contributional'etude des processus de la friture des aliments. Nutr Dieta 1977; 25: 112-9.

17. FAO/OMS. Grasas y aceites en la nutrición humana. Capítulo 6: Selección de usos de las grasas y de los aceites en la alimentación. Roma 1997.

18. Villalta J, Monferrer A. La fritura desde un punto de vista práctico (II). Aliment., Equipos Tecnol. 1993; 12(4): 85-90.

19. Dobarganes C, Marquez-Ruiz G. Interactions between fat and food during deep-frying. Eur J Lipid Sci Technol. 2000; 102:521-8.

20. Tirado DF, Acevedo D.A, Guzmán L.E. Freído por inmersión de los alimentos. Reciteia 2012: 72-80.

21. Valenzuela A, Sanhueza J, Nieto S, Petersen G, Tavella M. Estudio comparativo en fritura de la estabilidad de diferentes aceites vegetales. Aceites Grasas 2003; 53(4), 568-73.
22. Quiles JL, Ramirez- Tortosa MC, Yacoob P. Olive Oil and Health. CAB international, USA, 2006.

23. García MA, Ferrero $C$, Bertola $N$, Martino $M$, Zaritzky $N$. Edible coatings from cellulose derivatives to reduce oil uptake in fried products. Innov Food Sci Emerg Technol. 2002; 3:391-7.

24. Thanatuksorn P, Kajiwara K, Suzuki T. Characteristics and oil absorption in deep-fat fried batter prepared from ball milled wheat flour. J Sci Food Agric. 2010; 90:13-20.

25. Shih FF, Daigle KW. Oil uptake properties of fried batters from rice flour. J Agric Food Chem. 1999; 47:1611-5.

26. Shih F, Boue SM, Daigle KW, Shih BY. Effects of flour sources on acrylamide formation and oil uptake in fried batters. J Am Oil Chem Soc. 2004. 81:265-8.

27. Lamberg I, Hallstrom B, Olsson H. Fat uptake in a potato drying/frying process. Lebensm, Wiss, Technol. 1990; 23:295-300.

28. Krokida $M K$, Oreopolou $V$, Maroulis ZB, Marinos-Kouris D. Deep fat frying of potato strips-quality issues. Dry Technol. 2001; 19:879-935.

29. Rossel JB. Industrial Frying Process. GrasasAceites 1998; 49:282-95.

30. González CA, González CH. Globesidad y su posible componente infeccioso. Biosalud 2009; 8: 32 -42.

31. Gobierno de Chile. Ministerio de Salud. Encuesta Nacional de Consumo alimentario (ENCA). Disponible en http://web. minsal.cl/sites/default/files/ENCA.pdf

32. Carrillo L, Dalmau J, Martínez J, Solà R, Pérez F. Grasa de la dieta y salud cardiovascular. Nutr Clín Diet Hosp.2011; 31(2):6-25.

33. Mehta U, Swinburn B. A Review of Factors Affecting Fat Absorption in Hot Chips. Crit Rev Food Sci Nutr. 2001; 41:2, 133-54.

34. Torrejón $C$, Uauy R. Calidad de grasa, arterioesclerosis y enfermedad coronaria: efectos de los ácidos grasos saturados $y$ ácidos grasos trans. Rev Med Chil. 2011; 139(7):924-31.

35. Keys A, Anderson J, Grande F. Prediction of serum-cholesterol responses of man to changes in fats in the diet. Lancet 1957; 273: 959-66.

36. FAO/WHO. Joint FAO/WHO Expert Consultation on Fats and Fatty Acids in Human Nutrition (2008). Interim Summary of Conclusions and Dietary Recommendations on Total Fat \& Fatty Acids, Geneva. Disponibleen:http://www. who.int/nutrition/topics/FFA_summary_rec_conclusion.pdf

37. Kris-Etherton P, Yu S. Individual fatty acids on plasma lipids and lipoproteins: human studies. Am J Clin Nutr. 1997; 65: 1628S-44S.

38. Mozaffarian D, Katan M, Ascherio A, Stamler J, Willett W. Trans fatty acids and cardiovascular disease. N Engl J Med. 2006; 354: 1601-13.

39. Valenzuela A. Ácidos grasos con isomería trans, su origen y los efectos en la salud humana. Rev Chil Nutr. 2008; 35:162- 71.

40. Gamble $M H$, Rice P. Effect of pre-fry drying of oil uptake and distribution in potato crisp manufacture. Food $\mathrm{Sci}$ Technol. 1987; 22: 535-48.

41. Tutaa S, Palazoğlua TK, Gökmen V. Effect of microwave pre-thawing of frozen potato strips on acrylamide level and quality of French fries. J Food Eng. 2009; 97: 261-6.

42. Sanchez-Muniz FJ, Cuesta C. Tecnología de la Fritura: Alteraciones de las grasas utilizadas debido al proceso, tipo de grasa culinaria y alimento. Aliment., Equipos y Tecnol. 1991;3: 101-16. 